\title{
Monitoring and Analysing of Electric Data Basing on the Energy Internet in Laogang Solid Waste Recycling Base
}

\author{
Jianwen Nie ${ }^{1, \mathrm{a}}$, Xiaodong Wang ${ }^{1, \mathrm{~b}}$, Yuewei Chen ${ }^{1, \mathrm{c}}$, Guangcai Hao ${ }^{1, \mathrm{~d}}$, Yuzhao Hui ${ }^{2, \mathrm{e}}$, \\ Yiqi Lü, ${ }^{3, *}$, Da Xie ${ }^{3, g}$ \\ ${ }^{1}$ Shanghai Laogang Solid Waste Comprehensive Development co. Ltd, China \\ ${ }^{2}$ Shanghai environmental sanitation engineering design institute co. Ltd, China \\ ${ }^{3}$ Shanghai Jiaotong University, 800 Dongchuan RD. Minhang District, Shanghai, China \\ a niejianwen@hotmail.com, ${ }^{\mathrm{b}}$ wang_xd@smi-co.com, ${ }^{\mathrm{c}}$ cyw@smi-co.com, ${ }^{\mathrm{d}}$ hgc@smi-co.com, \\ eyuzhaohui36@163.com, ${ }^{\mathrm{f}} 597206911 @$ qq.com, ${ }^{\mathrm{g}}$ xieda@sjtu.edu.cn
}

Keywords: Energy internet, Cloud platform, Real-time monitoring, Recycling

\begin{abstract}
In order to make full use of the solid wastes, an energy internet is designed for Laogang base, the biggest place to recycle wastes in Shanghai. This energy internet combine heat and methane with electricity on the basis of current multi-energy system in Laogang to increase energy utilization rate and improve energy quality. For better supervisory control of the energy internet, intelligent monitoring devices which can process data from time to time are installed there. The data collected will be used to analyse the circumstance of factories or facilities after comprehensive calculation. In addition, a corresponding cloud platform has been built to display analytical graph to both Administrators and users so that expected response will be achieved.
\end{abstract}

\section{Introduction}

From partial electric network, to state grid, and then to smart power grid now, the development of the power grid has experienced a long period of time. Now that the stability and safety is guaranteed while using electricity, there are still many energy problems remain unsolved. As distributed energy resources emerging here and there, low efficiency of energy utilizing is becoming a much severer problem. Therefore, the concept of energy internet has been put forward $[1,2]$.

The purpose of energy internet is to coalesce different energies so that they can be produced, collected and dispatched together basing on both supply and demand side. While the energies are working together, scattered energy will not be ignored, the flow of energy will be arranged most reasonably. Thus, energy efficiency will be enhanced, and the idea of green energy will be promoted as well $[3,4]$.

Laogang solid waste recycling base has a multi-energy system with three kinds of energy: heat, methane and electricity. This base deals with $70 \%$ of household garbage in Shanghai and produce energy for itself and also other users outside the base. Because of the multi-energy situation and concentration of factories, it is quite appropriate to construct an energy internet, which will certainly improve the whole recycling process, in the base. Meanwhile, corresponding regulatory measures including intelligent monitoring device and cloud platform is now ready to serve $[5,6]$.

The main contribution of this paper:

(1) Design and partly implement the energy internet on the basis of current multi-energy system in Laogang base.

(2) Provide the comprehensive algorithm and the monitoring device to complete the monitoring and analyzing of the data from factories in the base.

(3) Construct a cloud platform to realize real-time monitoring and display the conclusion so that demand side response will be received. 


\section{Laogang Solid waste recycling base}

Laogang solid waste recycling base has four main parts: landfill site, waste incineration plant, leachate treatment plant, and biogas plant. First of all, the landfill deals with about 10,000 tons of wastes every day which can produce a huge amount of landfill gas and leachate. However, landfill is not a great choice to get rid of garbage, for it will take up a number of land resources. Hence waste incineration plant is necessary to turn wastes straightly into heat and electricity. Now the plant can incinerate over 3,000 tons of wastes every day, and further extension is on the schedule. Next, leachate treatment plant will use the leachate from landfill and incineration to produce marsh gas. Together with landfill gas, they will finally be stored or burned to become heat and electricity after purification.

\subsection{Energy Internet in Laogang}

Figure 1 shows the structure of energy internet designed for Laogang base. It connects the three energies and offers heat and electricity to other users or the grid while satisfying the operation of the base itself. The energy internet can change the independent situation of factories in the base, and thereby use resources more efficiently. Through P2G (Power to Gas) technology, the networks of electricity and gas are more closely related.
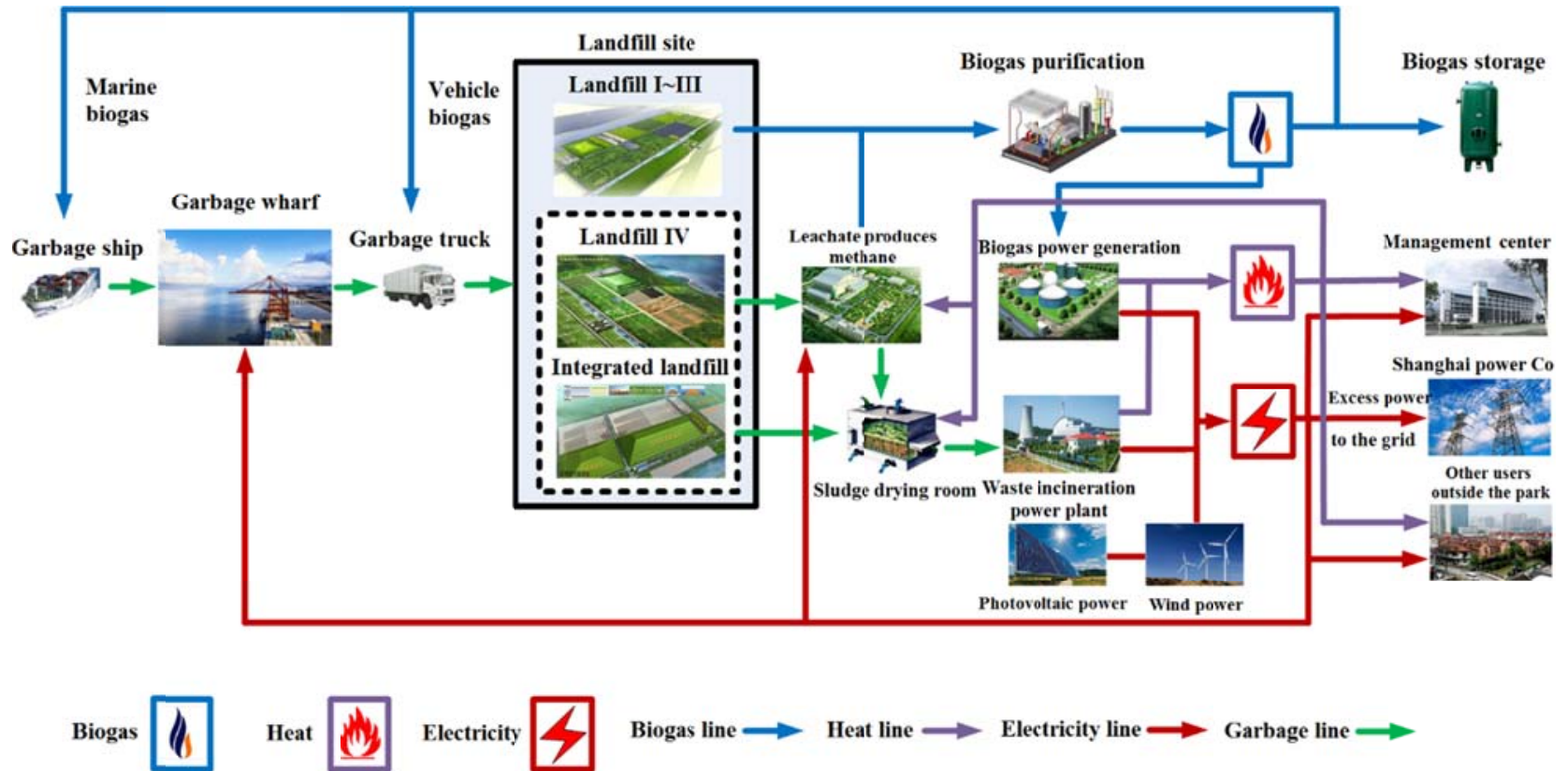

Figure 1 The Laogang base energy internet.

\subsection{Power Network}

Power network, which is the core framework of the energy internet, is also the key point for supervising and controlling its operation.

1. Generation Units

Overall, the generation units in the base mainly includes waste incineration plant, biogas plant, wind power plant and photovoltaic power plant. Incineration plant uses wastes as row material to generate power while recycling wastes. Biogas plant gathers landfill gas and marsh gas from leachate treatment plant to produce energy. Finally, wind power plant and photovoltaic power plant are renewable power generation units, which can solve the space problem of landfill. Among them, the construction of PV power plant is still planning.

\section{Utilization Units}

The internal production and living of the entire base will spend a lot of electricity, and leachate treatment plant uses the most to assist biological reaction to recycle pollutant. Most recycling process will consume much energy. For example, air blower, regulating pump and many large facilities do so. Garbage handling in garbage wharf also needs an amount of electricity. The rest 
energy in power network will be sold to Shanghai Power Company at a reasonable price after negotiation.

\section{Electric Parameters Monitoring}

As the most important energy form in the base, the monitoring and analyzing of electricity will help a lot in expanding and deepening the application of the energy internet, for it provides essential data for research on structure optimization or planning theory. After a period of time to collect data, the load variation and productivity law can be grasped, advice for power consumption and detection of aging apparatus can be made. The monitoring content is shown in Table 1.

Table 1 Monitoring point in the base.

\begin{tabular}{cccc}
\hline Type & No. & Position & \multicolumn{1}{c}{ Monitoring Quantities } \\
\hline \multirow{3}{*}{ Generation } & 1 & Waste Incineration Plant & Voltage, Current, Power, Frequency, Harmonic \\
Unit & 2 & Biogas Plant & Voltage, Current, Power, Frequency, Harmonic \\
& 3 & Wind Power Plant & Voltage, Current, Power, Frequency, Harmonic \\
& 4 & Photovoltaic power plant & Voltage, Current, Power, Frequency, Harmonic \\
\hline \multirow{3}{*}{ Utilization } & 1 & Leachate Treatment Plant & Voltage, Current, Power, Frequency, Harmonic \\
Unit & 2 & Garbage Wharf & Voltage, Current, Power, Frequency, Harmonic \\
& 3 & Management Center & Voltage, Current, Power, Frequency, Harmonic \\
& 4 & on-grid energy & Voltage, Current, Power, Frequency, Harmonic \\
\hline
\end{tabular}

\section{Calculating and Analysis method of the Data}

The intelligent monitoring device installed in the base uses AD7606, a 16 bit AD chip, for field collection. It will get the instantaneous value of three phase voltage and current for 256 times in $20 \mathrm{~ms}$. A complete sampling and calculating process takes $200 \mathrm{~ms}$ with $20 \mathrm{~ms}$ to sample, $180 \mathrm{~ms}$ to calculate. It will upload data to the cloud platform every 30s with 145 sampling and calculating process [7].

\subsection{Effective Value}

The calculation of effective value starts from its definition: it equals the amplitude of a direct current which generate the same heat as the alternating current in the same period. Therefore, the effective value of voltage and current will be calculated as follows:

$$
\begin{gathered}
U_{A(j)}=\sqrt{\frac{1}{T} \sum_{i=1}^{N} U_{a(i)}^{2} \times \frac{T}{N}}=\frac{1}{16} \sqrt{\sum_{i=1}^{256} U_{a(i)}^{2}} \\
I_{A(j)}=\sqrt{\frac{1}{T} \sum_{i=1}^{N} I_{a(i)}^{2} \times \frac{T}{N}}=\frac{1}{16} \sqrt{\sum_{i=1}^{256} I_{a(i)}^{2}}
\end{gathered}
$$

Where $\mathrm{T}$ is eliminated and $\mathrm{N}$ equals the times data is collected in one period.

\subsection{Power}

Power includes active power, reactive power and apparent power. Active power shows the amount of electricity converted into other energies, which can be calculated through three-meter method:

$$
P_{\mathrm{i}}=P_{a(i)}+P_{b(i)}+P_{c(i)}=U_{a(i)} I_{a(i)}+U_{b(i)} I_{b(i)}+U_{c(i)} I_{c(i)}
$$

Then, the active power in one sampling and calculating process can be calculated: 


$$
P=\frac{\sum_{i=1}^{N} P_{i} \times \Delta t}{N \times \Delta t}=\frac{\sum_{i=1}^{256} P_{i}}{256}
$$

Analogously, reactive power still uses three-meter method, but unlike active power, its calculation must put phase angle difference into consideration. Therefore, the sample sequence of current is delayed for $1 / 4$ cycle, and then apply equation (3) and (4):

$$
\begin{array}{r}
Q_{i}=U_{a(i)} I_{a(i+64)}+U_{b(i)} I_{b(i+64)}+U_{c(i)} I_{c(i+64)} \\
Q_{i}^{\prime}=U_{a(i)} I_{a(i-192)}+U_{b(i)} I_{b(i-192)}+U_{c(i)} I_{c(i-192)} \\
Q=\frac{\left(\sum_{i=1}^{192} Q_{i}+\sum_{i=193}^{256} Q_{i}\right) \times \Delta t}{256 \times \Delta t}=\frac{\sum_{i=1}^{192} Q_{i}+\sum_{i=193}^{256} Q_{i}}{256}
\end{array}
$$

Apparent power is the maximum value of active power that the equipment can reach. Its value equals the product of the effective value of voltage and current. Thus, by using equation (1) and (2), the calculation of apparent power can be deduced:

$$
S=\frac{\sqrt{\sum_{i=1}^{N} U_{a(i)}^{2} \cdot \sum_{i=1}^{N} I_{a(i)}^{2}}+\sqrt{\sum_{i=1}^{N} U_{b(i)}^{2} \cdot \sum_{i=1}^{N} I_{b(i)}^{2}}+\sqrt{\sum_{i=1}^{N} U_{c(i)}{ }^{2} \cdot \sum_{i=1}^{N} I_{c(i)}^{2}}}{N}
$$

\subsection{Power Factor}

Power factor is extensively used in inspecting practical efficiency of electrical equipment. In practical production, power factor should be kept as high as possible. Its calculation is not simply $\mathrm{P} / \mathrm{S}$, for there may be distortion power contributing to apparent power. So the equation $S^{\prime 2}=P^{2}+Q^{2}+T^{2}$ must be used to find the exact apparent power, and calculate the power factor as follows:

$$
\cos \varphi=P / S^{\prime}
$$

\subsection{Power Consumption}

Power consumption is the time-domain integral of power. Take active power as an example, if $P_{i}$ is the average power in $30 \mathrm{~s}$, then the power consumption in this $30 \mathrm{~s}$ is $\frac{P_{i}}{120}$ with unit $k W \cdot h$. From this point, the consumption within any period can be calculated:

$$
E_{p}=\sum_{i=1}^{n} \frac{P_{i}}{120}
$$

\subsection{Frequency and Harmonic}

To calculate frequency and harmonic, Fast Fourier Transform (FFT) is need at the first place. After FFT, the harmonic amplitude and phase angle will be figured out. By using the phase angle and convert it into frequency, it is likely to calculate the frequency for one 30 seconds:

$$
f=50+\frac{\sum_{i=1}^{145}\left(\varphi_{i+1}-\varphi_{i}\right)}{0.4 \pi \times 145}
$$


Where $\varphi_{i}$ represents the phase of one sampling and calculating period.

To measure the effect of harmonics, the amplitudes are essential. THD and VCF are usually used for quantification of the size of harmonics. THD is total harmonics distortion, while VCF refers to waveform deviation factor. Both the two parameters can reveal the condition of harmonics:

$$
\begin{gathered}
\text { THD }=\frac{\sqrt{\sum_{i=1}^{50} G_{i}^{2}}}{G_{1}} \\
V C F=\frac{\sum_{i=2}^{50} G_{i}}{G_{1}}
\end{gathered}
$$

Where $G_{i}$ stands for the amplitude of order $i$ harmonic. If these two parameters are big, it means the harmonic is seriously affecting the power quality, countermeasures must be made as soon as possible.

\section{The Application on Cloud Platform}

Through three layers architecture of cloud: IaaS, PaaS and SaaS, a cloud platform is built to realize the real-time monitoring and analyzing of the energy internet in Laogang.

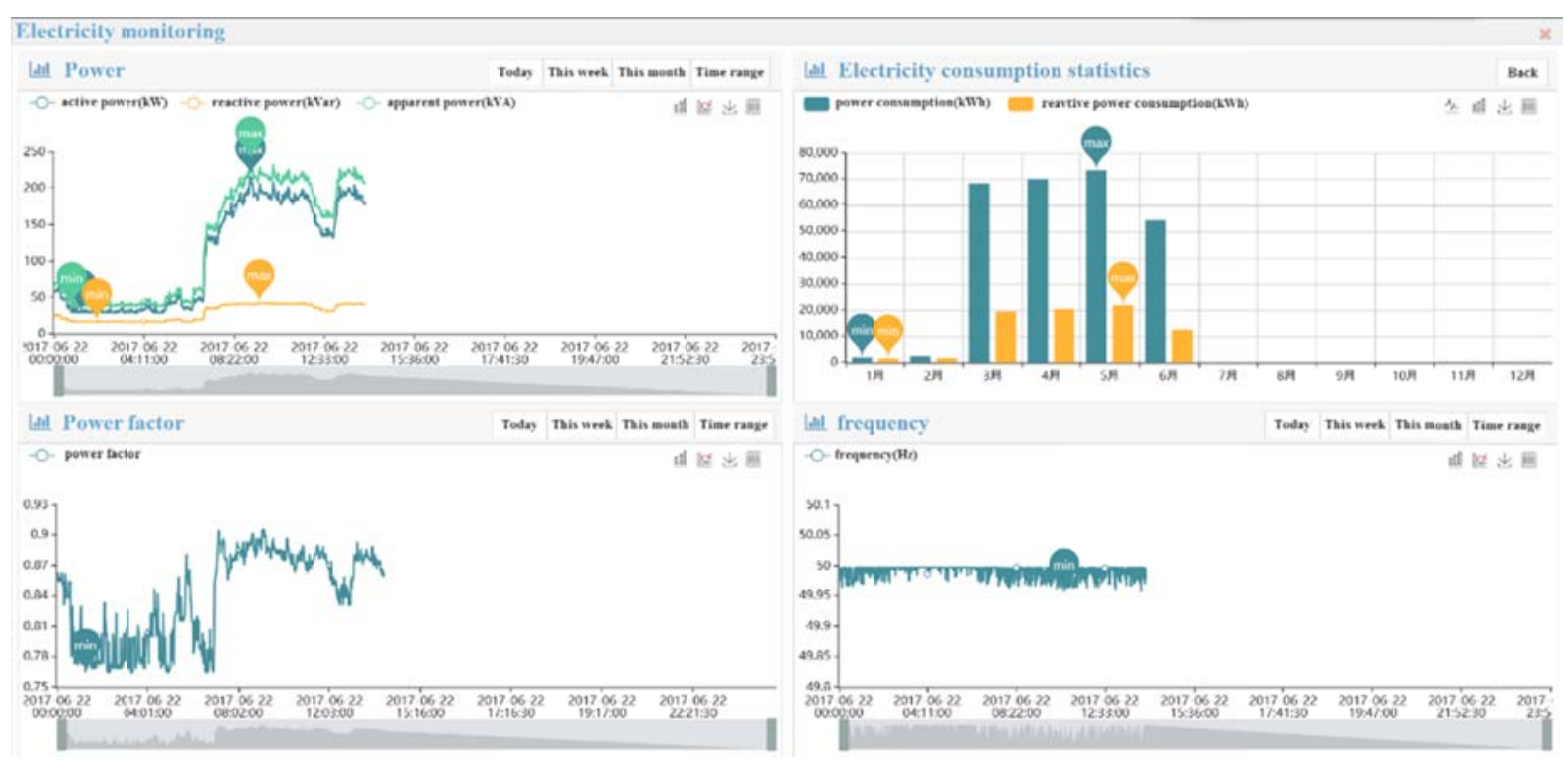

Figure 2 Power monitoring interface.

The real-time monitoring part includes power, bus voltage, transformer temperature, frequency and so on. Along with the uploading, the real-time data on the platform will update every 30s. An example is power monitoring interface as shown in Figure 2. It consists of power diagram, power factor diagram, power consumption diagram and frequency diagram. Both real-time and historical data are included in these interfaces to give the users a basic idea of the operating condition of the energy internet in the base.

The analyzing part aims at demand side response with six sections: total electricity statements, power analysis, power losses analysis, space-time balance of loads, power quality and systemic analysis. Figure 3 shows the harmonic analysis interface which is a portion of power quality. There are the ratios of the harmonics, spectrogram and analysis curve in the interface. For different order of harmonics, there are specific harmonic elimination method. The improvement of Power quality will not only improve stability and reliability of equipment, but also satisfy both demand and supply side. There are also prediction for power consumption depending on weather and historical data, or 
benchmarking on the cloud platform. These function will provide a technical reference for the users.

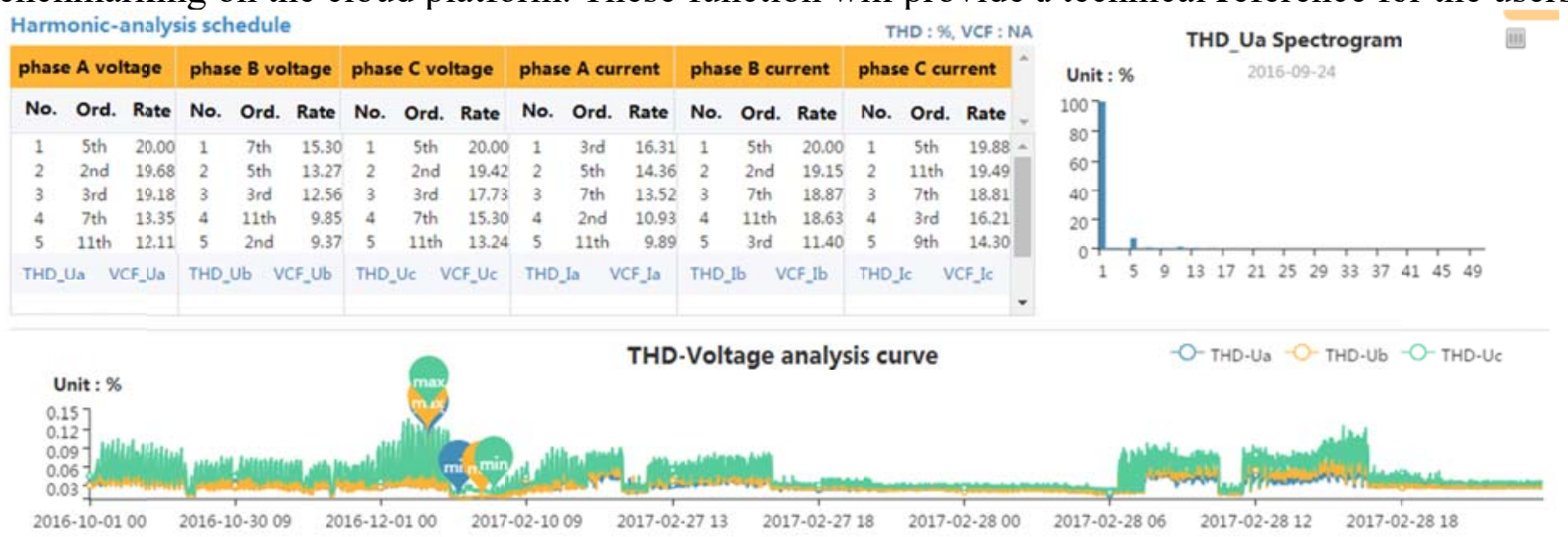

Figure 3 Power analysis interface.

\section{Summary}

Basing on the multi-energy system in Laogang, it is appropriate to build an energy internet demonstration base. Now the bulk of the construction has been finished. Although the energy internet in Laogang base is still not functioning very well, the recycling and reutilization of different kinds of energy resources is already benefiting the base. It is likely that the new operational mode will provide some experience for larger and more practical energy internet.

Also, the monitoring and analyzing method and the cloud platform is an effective assistance for the energy internet. Now the calculation will reveal the information on electricity usage comprehensively, and it has been examined that the data algorithm is accurate, and the monitoring device and cloud platform are reliable and stable.

However, time is still needed to accumulate data so that there will be enough information for the big data analysis. It is predictable that an ideal energy internet which combines supply and demand side will be realized in the near future.

\section{References}

[1] S Tian, W Luan, D Zhang, C Liang, Y Sun. Technical Form and Key Technology of Energy Internet [J]. Proceedings of the CSEE, 2015, (14):3482-3494.

[2] T Yan, H Cheng, P Zeng, Z Ma, L Zhang, S Tian. Architecture and Key Technology of Energy Internet [J]. Power System Technology, 2016, (01):105-113.

[3] H Sun, Q Guo, S Pan, J Wang. Energy Internet: Driver, Review and Outlook [J]. Power System Technology, 2015, (11):3005-3013.

[4] J Hong, J Liu, Y Xiang, Y Niu. Preliminary Understanding and Research Prospect of City Energy Internet [J]. Electric Power Automation Equipment, 2017, (06):15-25.

[5] X Hu, M Zhang, J Yu, G Zhang. Current Situation, Problems and Countermeasures of Chinese Kitchen Waste [J]. Acta Ecologica Sinica, 2012, (14):4575-4584.

[6] J Wang, W Sheng, J Wang, H Yang, Q Song. Design and Realization of Unified Data Acquisition and Monitoring System for Low Voltage Distribution Network [J]. Automation of Electric Systems, 2012, (18):72-76+81.

[7] R Maidstone, T Hocking, G Rigaill, et al. On optimal multiple change point algorithms for large data, Statistics \& Computing. 27 (2017) 519-533. 